\title{
Experience with carboplatin and etoposide maintenance chemotherapy in patients with extensive stage small cell lung cancer
}

\author{
Amaan Siddiqi' \\ Huzefa Bahrain ${ }^{2}$ \\ Michael Auerbach ${ }^{1,2}$ \\ 'Georgetown University School \\ of Medicine, ${ }^{2}$ Auerbach Hematology \\ and Oncology, Baltimore, MD, USA
}

This article was published in the following Dove Press journal:

Lung Cancer:Targets and Therapy

26 August 2011

Number of times this article has been viewed

\begin{abstract}
Purpose: To determine whether maintenance therapy with carboplatin and etoposide improves progression-free and overall survival in patients with extensive stage small cell lung cancer, compared to the standard four to six cycles of cisplatin and etoposide.

Methods: Forty-two patient records (25 males and 17 females) were retrospectively reviewed in a single community practice. All patients were over the age of 18 , with pathologically and radiographically proven extensive stage small cell lung carcinoma (SCLC). The starting doses of chemotherapy were carboplatin, AUC (area under the curve) of 6 IV day 1, and etoposide, $100 \mathrm{mg} / \mathrm{m}^{2}$ IV days $1-3$. The regimen was administered every 3 weeks and increased to every 4 to 5 weeks as tolerated or until documented progression occurred. Varying second-line chemotherapies were used.

Results: Median overall survival was 17 months from diagnosis, with a progression-free survival of 15 months. Seventy-nine percent of the patients survived more than 10 months. The 1 - and 2-year overall survival (OAS) rates were 0.74 (31 patients) and 0.31 (13 patients), respectively. The 1- and 2-year progression free survival (PFS) rates were 0.50 (21 patients) and 0.21 ( 9 patients), respectively.
\end{abstract}

Conclusion: The improved overall and progression-free survival compared to the current standard in this small single center cohort suggests that maintenance therapy with carboplatin and etoposide to progression may be a prudent area for further investigation in a properly powered randomized, controlled trial.

Keywords: progression-free survival, maintenance therapy, overall survival, metastasis

\section{Introduction}

Lung cancer is the leading cause of cancer-related death in the US. Small cell lung cancer (SCLC) accounts for $15 \%$ of all cases, with tobacco use present in nearly all cases. The annual incidence of SCLC ranges from 22,000 to 34,000 cases per year. ${ }^{1}$ SCLC is often associated with paraneoplastic syndromes, most notably Lambert-Eaton myasthenic syndrome, SIADH, and ectopic ACTH production. ${ }^{2}$ In addition, SCLC has a tendency to metastasize early in the disease, with many patients presenting with metastasis at the time of diagnosis. ${ }^{3}$

Recognized management options include radiation therapy and chemotherapy, depending on disease stage. In rare circumstances, surgery may be employed, but due to the rapid growth and dissemination of SCLC, surgery as primary therapy is largely proscribed. ${ }^{4}$ Treatment strategies most often focus on systemic therapy, as SCLC is responsive to chemotherapy. Concurrent radiation is used if disease is limited to the thorax within a single radiation port, with 5-year survival rates of 5\%.
Correspondence: Amaan Siddiqi

91 10 Philadelphia Road,

Suite $3 \mid 4$, Baltimore,

MD 21237, USA

Tel +l 4107804050

$\mathrm{Fax}+\mathrm{I} 4107804060$

Email amaan.siddiqi@gmail.com
Lung Cancer:Targets and Therapy 20I I:2 4I-45

(C) 20I I Siddiqi et al, publisher and licensee Dove Medical Press Ltd. This is an Open Access article

Dovepress

http://dx.doi.org/10.21 47/LCTT.S22864 which permits unrestricted noncommercial use, provided the original work is properly cited. 
Staging is divided into limited and extensive stage disease. Extensive stage disease extends beyond a single radiation port (from the ipsilateral hemithorax to the contralateral hemithorax, or to distant sites). Untreated patients with extensive disease have a median survival of 6 weeks. ${ }^{1}$ The current standard for extensive stage SCLC is combination chemotherapy, using etoposide plus a platinum derivative (often cisplatin) for four to six cycles, or two cycles past maximum response. ${ }^{1}$ In fact, treatment with cisplatin plus etoposide has been standard for over 20 years, as few other therapies have been shown to improve survival. Sixty to eighty percent of extensive stage disease responds to chemotherapy, with only $15 \%-20 \%$ achieving complete remission. ${ }^{3}$

Myelosuppression, nausea, vomiting, peripheral neuropathy, fatigue, and nephrotoxicity, are the most common toxicities associated with this chemotherapy regimen. Due to the cumulative toxicity of the cisplatin, patients can usually tolerate only a limited number of cycles. Median survival is 9-10 months using combination chemotherapy (cisplatin/ etoposide), with few patients surviving past 2 years. ${ }^{3}$ Since carboplatin has a more favorable toxicity profile with likely equal efficacy, it is commonly used as an alternative to cisplatin. Carboplatin, while better tolerated, does cause more myelosuppression. .,3,6 $^{1}$

The increasing use and clinical efficacy of 5HT3receptor antagonists and substance $\mathrm{P}$ antagonists over the years, such as ondansetron, granisetron, and aprepitant, have led to an improvement in symptoms of nausea and vomiting. ${ }^{7}$ The approval and use of growth factors has led to decreased morbidity caused by cytopenias, making treatment interruption less common. The strategy of maintenance chemotherapy has demonstrated improved survival in published series. A literature review by Sculier et al evaluating the effectiveness of maintenance chemotherapy used in several randomized trials (in varying stages of disease) showed mixed results, though none of the regimens used carboplatin. Significant improvements in survival were seen in patients with limited stage disease treated with maintenance cisplatin/etoposide, though the regimen was not used in patients with extensive stage disease. ${ }^{8}$ Similarly, in a meta-analysis by Bozcuk et al, improvements in both 1- and 2-year overall survival were seen with maintenance chemotherapy, although carboplatin/etoposide was not one of the regimens analyzed. ${ }^{9}$

The purpose of this retrospective analysis is to determine whether maintenance therapy with carboplatin plus etoposide given every 3 to 5 weeks until progression or death resulted in improvements in overall and progression-free survival in the study group.

\section{Patients and methods}

Forty-two eligible patients' charts were reviewed for the study. Consecutive, nonselected, carboplatin/etoposide treated patients meeting criteria for extensive stage SCLC were included in the analysis. Inclusion was restricted to patients with pathologically proven SCLC and radiographically proven extensive stage disease, who were treated with the intention of continuous maintenance therapy with carboplatin and etoposide, until progression or death. No exclusions for age, race, or performance status were present. ECOG scores were used to assess performance status at the time of diagnosis (Table 1). Exclusions included limited stage disease, complete data unavailable, or diagnosis inconsistent with SCLC. Demographic data was compiled (Table 2).

Time to progression and overall survival were calculated. The starting doses of chemotherapy were an AUC of 6 for carboplatin on day 1 and $100 \mathrm{mg} / \mathrm{m}^{2}$ of etoposide on days $1-3$.

Chemotherapy was then administered every 3 weeks initially. Dose reductions of up to $25 \%$ or prolongation of treatment interval to 4 or 5 weeks was done as needed for myelosuppression. All patients receiving maintenance chemotherapy required dose reduction for grade III or IV neutropenia or thrombocytopenia. Treatment was continued until documented radiographic progression occurred. All underwent serial CT scanning for known sites of disease. All received prophylactic cranial irradiation. Sixteen were placed on second-line chemotherapy, with three receiving third-line treatment. For the purpose of this study, progression-free survival only applies to first-line therapy.

\section{Data collection}

Names and medical records were recorded on a separate spreadsheet. The following information was collected: age, gender, date of diagnosis, date of initial treatment, chemotherapy regimen, frequency of treatment, secondand/or third-line treatment, performance status, presence

Table I Performance status

\begin{tabular}{ll}
\hline ECOG score & Number of patients \\
\hline 0 & 8 \\
1 & 17 \\
2 & 12 \\
3 & 5 \\
\hline
\end{tabular}


Table 2 Demographics

\begin{tabular}{ll}
\hline & Number (\%) \\
\hline Male & $25(59.5)$ \\
Female & $17(40.5)$ \\
Caucasian & $41(97.6)$ \\
African-American & $\mathrm{I}(2.4)$ \\
Other & $0(0)$ \\
Mean age at diagnosis & 62.5 years \\
\hline
\end{tabular}

and location of metastasis, date of metastasis, and date of progression or death.

\section{Data analysis}

Data are presented as mean, median, and range. One and 2-year overall survival and progression-free survival were also calculated.

\section{Results}

Forty-two patients, 25 males and 17 females, were included in the analysis. Forty-one were Caucasian, and one was AfricanAmerican. The median age at diagnosis was 62.5 years with a range of 44-85 years. The earliest diagnosis was January 1994; the most recent August 2006. At the time of diagnosis, performance status was assigned, using the ECOG scoring system. Sites of metastasis were noted, with seven patients having multiple sites of metastasis (Table 3 ).

The median overall survival was 17 months from time of diagnosis, with a range of 2-80 months. The mean overall survival was 22.5 months. One- and 2-year overall survival rates were 0.74 (31 patients) and 0.31 (13 patients), respectively.

The median progression-free survival was 15 months from diagnosis, with a range of $2-80$ months. The mean progression free survival was 20.4 months. One- and 2-year progression free survival rates were 0.55 (23 patients) and 0.26 (11 patients), respectively. Survival data are presented in Table 4.

\section{Discussion}

The use of cisplatin and etoposide has been proven to be an effective regimen for extensive stage SCLC, resulting in

\section{Table 3 Metastasis}

\begin{tabular}{ll}
\hline Sites of metastasis & Number of patients \\
\hline Liver & 13 \\
Brain & 11 \\
Bone & 10 \\
Multiple sites of metastasis & 7
\end{tabular}

Note: Items are not mutually exclusive. improved survival for patients who receive four to six cycles of therapy. Carboplatin is often used in place of cisplatin, due to its more favorable side effect profile, while maintaining efficacy. ${ }^{1,3,6}$ While the efficacy of carboplatin plus etoposide in extensive stage SCLC is well established, maintenance therapy is controversial, and would be difficult if cisplatin were used instead. With improved second generation chemotherapy and better palliative agents, maintenance chemotherapy is better tolerated. The results from the previously published trials examining the benefit of maintenance are unclear with positive results seen only in certain subsets studied. ${ }^{8,9}$

A previously published clinical trial using a limited number of cycles of cisplatin and etoposide, yielded a median survival of 9-10 months, consistent with the published norm. ${ }^{3}$ With a range from 2 months up to 80 months of overall survival times from diagnosis in this study, median survival was calculated so as to limit the influence of outliers. In fact, there were only nine $(21.4 \%)$ who survived fewer than 10 months. Thirty three patients (78.6\%) exceeded the previously documented median survival time of approximately 10 months. Thirteen were living 2 years after diagnosis, with eleven of those progression-free at 2 years. Four were still alive at the time of this study, and appeared free of progression. Eleven (26.2\%) developed brain metastases.

Previous studies analyzing chemotherapy without maintenance show 1- and 2-year overall survival (OS) to be 0.30 and 0.10 , respectively. ${ }^{9}$ In our study, improvements in both 1 -year OS (30\% to $74 \%)$, and 2 -year OS (10\% to $31 \%)$ were seen. In addition, previous studies showed progression-free survival (PFS) at 1 and 2 years to be 0.13 and 0.10 , respectively, for nonmaintenance treatment. ${ }^{9}$ In this analysis, improvements were seen in 1-year PFS (13\% to 55\%), and 2 -year PFS (10\% to $26 \%$ ). One to 50 sets of seven cycles of carboplatin and etoposide were administered. Thirty-nine patients responded to first-line therapy. All patients who responded to treatment eventually required dose reduction. Dose reduction was most commonly required after cycle number eight. Myelosuppression was the most common limiting toxicity. All other toxicities were manageable with supportive care or were relatively mild.

Sixteen of the 42 patients were placed on second-line chemotherapy. Six were placed on paclitaxel alone, four on topotecan alone, and one on doxorubicin. Three were placed on the combination of topotecan and navelbine, one on topotecan and gemcitibine, and one on a combination of topotecan and paclitaxel. Three received third-line therapies. 
Table 4 Survival data

\begin{tabular}{|c|c|c|c|c|c|c|}
\hline & \multicolumn{3}{|c|}{ Progression-free survival (PFS) } & \multicolumn{3}{|c|}{ Overall survival (OAS) } \\
\hline & $<10$ months & 10-24 months & 24 months & $<10$ months & I0-24 months & 24 months \\
\hline Patients $(n=42)$ & $13(30.9)$ & $18(42.9)$ & II (26.2) & $9(2 \mid .4)$ & $20(47.6)$ & $13(30.9)$ \\
\hline
\end{tabular}

Note: Data are presented as no. (\%) unless otherwise indicated.

Of these three, one received taxotere, another topotecan, and the third gemcitibine.

All 42 patients received prophylactic cranial irradiation (PCI). The increase in overall and progression-free survival may be partially attributed to the PCI, which has been shown to increase overall survival in limited and extensive stage small cell lung cancer. ${ }^{10,11}$ With the significant increase in survival seen in this study, it is postulated that maintenance chemotherapy confers an additional benefit.

With a median overall survival rate of 17 months (and 1 -year and 2-year PFS of 55\% and 26\%, respectively) in this study, maintenance chemotherapy appears to offer a survival benefit compared to the standard four to six cycles of cisplatin and etoposide. These findings support previously published evidence suggesting that maintenance chemotherapy provides considerable improvement over the current standard in patients with extensive stage SCLC. ${ }^{6}$

Two previous studies demonstrated the efficacy of maintenance therapy in SCLC. ${ }^{2}$ In 1998, Sculier et al analyzed 13 randomized trials, in which patients were treated with maintenance regimens involving various chemotherapeutic agents. Chemotherapies analyzed include cisplatin/etoposide in limited stage disease, and various combinations of cyclophosphamide, vincristine, methotrexate, doxorubicin, and vindesine. Though none of the regimens used were carboplatin/etoposide in extensive stage disease, evidence of benefit with maintenance was seen. Three of the 13 studies showed significant increases in overall survival, with one study showing a significant decrease. ${ }^{8}$

In 2005, Bozcuk et al performed a meta-analysis of 14 clinical trials, some of which were also included in Sculier et al's study. Again, various regimens were used in either limited stage or extensive stage disease, though the cisplatin/etoposide regimen was only evaluated in limited stage disease. The therapies assessed included cisplatin/ etoposide in patients with limited stage disease, in addition to topotecan or etoposide alone, and various combinations of cyclophosphamide, vincristine, doxorubicin, lomustine, methotrexate, vindesine, and hexamethylmelamine. ${ }^{9}$ The meta-analysis by Bozcuk et al showed that maintenance chemotherapy modestly improved 1-year overall survival (30\% to $39 \%$ ) and 2 -year overall survival (10\% to $14 \%$ ), as compared to standard chemotherapy. Improvements in 1-year progression-free survival (13\% to $23 \%$ ), as well as 2 -year progression-free survival (10\% to $13 \%$ ) were observed as well. ${ }^{8}$ Although neither Sculier et al nor Bozcuk et al analyzed use of maintenance carboplatin/etoposide in extensive stage SCLC, both meta-analyses provided rationale for future use of maintenance chemotherapy. Of note, both publications stressed the need for future large, randomized trials of good quality to assess the use of maintenance therapy. ${ }^{8,9}$

There are potential concerns with our study. With a sample population size of only 42 patients, the study is not well-powered, and effectively, this is a single institution study without a control group. Also, this study was neither randomized nor blinded, both limitations of retrospective studies. Despite these drawbacks, this study demonstrates a nearly two-fold improvement compared to the published results with the current standard. As a result of these encouraging findings, further study into chemotherapy with carboplatin and etoposide to progression in extensive stage SCLC appears warranted.

\section{Conclusion}

Through a retrospective analysis, we have shown that the efficacy of maintenance chemotherapy with carboplatin and etoposide may exceed that of standard cisplatin and etoposide for four to six cycles in extensive stage SCLC. Both overall survival and PFS were significantly longer in this series than is currently described with standard of care. Although we acknowledge that a retrospective study without randomization, blinding, or control groups is not significant enough to change current guidelines, the patients in this study were consecutive and nonselected, and the results found are particularly encouraging. Because of these findings, as well as previous anecdotal evidence, further study using randomized, controlled trials should be pursued. We also suggest this tactic of maintenance therapy be considered an alternative to the current standard of care in the future.

\section{Acknowledgment}

We acknowledge the staff at Auerbach Hematology and Oncology for their valuable assistance. 


\section{Disclosure}

None of the authors have any relevant financial disclosures.

\section{References}

1. El Maalouf G, Rodier JM, Faivre S, et al. Could we expect to improve survival in small cell lung cancer? Lung Cancer. 2007; 57(Suppl 2):S30-S34.

2. Payne M, Bradbury P, Lang B, et al. Prospective study into the incidence of Lambert Eaton myasthenic syndrome in small cell lung cancer. J Thorac Oncol. 2010;5(1):34-38.

3. Demedts IK, Vermaelen KY, van Meerbeeck JP. Treatment of extensivestage small cell lung carcinoma: current status and future prospects. Eur Respir J. 2010;35(1):202-215.

4. Sher T, Dy GK, Adjei AA. Small cell lung cancer. Mayo Clin Proc. 2008;83(3):355-367.

5. Bayman NA, Sheikh H, Kularatne B, et al. Radiotherapy for smallcell lung cancer-Where are we heading? Lung Cancer. 2009;63(3): 307-314.
6. Simon GR, Turrisi A. American College of Chest Physicians Management of small cell lung cancer: ACCP evidence-based clinical practice guidelines (2nd edition). Chest. 2007;132(Suppl 3): 324S-339S.

7. Billio A, Morello, Clarke MJ. Serotonin receptor antagonists for highly emetogenic chemotherapy in adults. Cochrane Database Syst Rev. 2010;1:CD006272.

8. Sculier JP, Berghmans T, Castaigne C, et al. Maintenance chemotherapy for small cell lung cancer: a critical review of the literature. Lung Cancer. 1998;19(2):141-151.

9. Bozcuk H, Artac M, Ozdogan M, et al. Does maintenance/consolidation chemotherapy have a role in the management of small cell lung cancer (SCLC)? A metaanalysis of the published control trials. Cancer. 2005; 104(12):2650-2657.

10. Slotman B, Faivre-Finn C, Kramer G, et al. Prophylactic cranial irradiation in extensive small-cell lung cancer. $N$ Engl $\mathrm{J}$ Med. 2007;357(7):664-672.

11. Patel S, Macdonald OK, Suntharalingam M. Evaluation of the use of prophylactic cranial irradiation in small cell lung cancer. Cancer 2009;115(4):842-850.

\section{Publish your work in this journal}

Lung Cancer: Targets and Therapy is an international, peer-reviewed, open access journal focusing on lung cancer research, identification of therapeutic targets and the optimal use of preventative and integrated treatment interventions to achieve improved outcomes, enhanced survival and quality of life for the cancer patient. Specific topics covered in the journal include:

\section{Dovepress}

Epidemiology, detection and screening; Cellular research and biomarkers; Identification of biotargets and agents with novel mechanisms of action; Optimal clinical use of existing anticancer agents, including combination therapies; Radiation and surgery; Palliative care; Patient adherence, quality of life, satisfaction; Health economic evaluations.

Submit your manuscript here: http://www.dovepress.com/lung-cancer-targets--therapy-journal 